\title{
Oral Health Determinants and Demographic Factors of Permanent Tooth Extraction in Iranian Adults
}

\author{
Lida Jarahi' ${ }^{1}$ Neda Jarahi ${ }^{2}$, Maryam Emadzadeh ${ }^{3}$ \\ ${ }^{1}$ Addiction Research Center, Faculty of Medicine, Mashhad University of Medical Sciences, Mashhad, Iran \\ ${ }^{2}$ Department of Dental Prosthesis, Faculty of Dentistry, Mashhad University of Medical Sciences, Mashhad, Iran \\ ${ }^{3}$ Department of Community Medicine, Faculty of Medicine, Mashhad University of Medical Sciences, \\ Mashhad, Iran \\ Email: *Jarahil@mums.ac.ir
}

Received 24 December 2015; accepted 14 March 2016; published 17 March 2016

Copyright (C) 2016 by authors and Scientific Research Publishing Inc.

This work is licensed under the Creative Commons Attribution International License (CC BY). http://creativecommons.org/licenses/by/4.0/

(c) () Open Access

\begin{abstract}
Preservation of teeth until old ages indicates oral health status. Improving life expectancy in developing countries makes teeth care an important topic. This study assessed reasons of tooth extraction (TE), oral health determinants and demographic factors of permanent TE in Iranian adults. Adult people who referred to dental clinic of Mashhad Medical University in 2012 for dental extraction and lived in Mashhad city, participated in this study by convenient sampling method after obtaining informed consent. Demographic characteristics, oral health status, health habit and reasons of TE of participants were assessed. Data were analyzed by SPSS11.5 by Chi-Square, T-Test, ANOVA, Mann-Whitney and Kruskall-Wallis tests. Totally 254 people participated (383 extracted teeth) that $55 \%$ were female. Mean age of participants was 39.3 years; females were 6.1 years younger than males. The main reason of TE was dental caries $(55.1 \%)$, following by impaction. There was significant difference between average times of tooth brushing in both sexes, but difference was not shown in sugar intake. Except in people with college degrees that dental caries is the main cause of TE. With increase in life expectancy to more than $\mathbf{7 0}$ years old in Iranian, the age of TE especially in women is younger than expected. The most common cause of TE is dental caries that seem in spite of improving health indexes in Iran, poor oral hygiene remains as a problem. There is no significant difference between frequency of sugar intake and level of education that indicates unhealthy dietary habits related to tooth decay.
\end{abstract}

Keywords

Oral Health Determinant, Tooth Extraction, Demographic Factor, Mashhad, Iran

${ }^{*}$ Corresponding author. 


\section{Introduction}

Poor oral hygiene was highlighted as a serious public health problem in 2010 WHO report about "equity, social determinants and public health programs” [1]. About 3.9 billion people suffered from oral diseases in 2010 and permanent teeth caries, with prevalence of 35\%, were the most common global burdens of diseases in all ages [2]. Life expectancy is one of the most important measures that are improved in developing countries nowadays, so quality of life improvement is one of the most interesting topics of policymakers. Life expectancy improvement has been made teeth care an important topic, as it is shown that this index will be higher in countries with people having more teeth, and tooth preservation was accompanied by higher quality of life [3] [4]. In recent years, changes in life style and food habits, higher consumption of sweetened and acidic beverage, together with much use of whitening toothpastes, have been led to more tooth enamel erosion that indicates the importance of tooth extraction (TE) evaluation, tooth care and its relation with life expectancy in public health [5]-[7].

According to impressive improvement in dental treatment, preservation of permanent teeth until old ages is not unachievable, and TE is just considered as a final treatment. Nevertheless, in less developed countries or in countries which people are not under assurance, TE statistics, especially for treatable teeth, are high [8]. Preservation of tooth and its supporting tissue until old ages indicates both oral health level and health system efficacy. In other side, some patients are not available to repair decayed teeth just because of financial issues, which show patients need to health system supports [9].

People with low socioeconomic status, have lower access to dental cares, despite they have more tooth complications. So many researches imply different behavioral and socioeconomic determinants in teeth loss [9] [10]. Smoking cessation and higher access to dental care lead to better dental preservation, and using tobacco, lower socioeconomic status, financial problems and underlying diseases accompany higher dental caries, lead to more teeth extraction [11] [12]. Improving education and hygiene level have been shown as important as developing dental services such as personnel, Technique and materials [13].

Knowledge about causes and risk factors of permanent TE, determines its preventable causes and demographic factors will help us to plan new educational approaches. This study has assessed reasons of tooth extraction, oral health determinants and demographic factors of permanent TE in Iranian adults, among people who referred to dental clinic of Mashhad University.

\section{Methods}

Adult people who lived in Mashhad city and have referred to dental clinic of dentistry faculty of Mashhad University of Medical Sciences participated in this cross-sectional study after obtaining informed consent. Sample size was determined 250 participants based on prevalence of dental caries in similar studies with $P=60 \%$ and alpha $=0.05$ [14]. Participants enter the study in convenient sampling method. In Mashhad school of dentistry, patients make an appointment by using a phone call and interaction voice response, so the investigator did not interfere in patients choosing process.

A trained interviewer asked participants; characteristics and habits like age, sex, education, employment, number of children, underlying diseases, addiction, drug history, oral health status, health habits such as frequency of tooth brushing per day and reasons of tooth extraction. Type and number of extracted teeth, upper/lower jaw and tooth number were also noted. According to the investigator's diagnosis, oral health status was categorized as poor, moderate and high status.

Observation for determination reasons of TE was performed and checklist fulfilled by investigator. Oral health status was observed by dental mirror and was categorized to three groups good, moderate and poor as follow manner:

1) Cervical calculus more than 3 millimeters height was categorized in group poor oral health.

2) Cervical calculus between 1 to 3 millimeters height moderate but if this status was with moderate or severe gingivitis it categorized in group poor oral health.

3) No calculus or existence of linear calculus in the cervical location of teeth with less than one millimeter height was categorized good, but if this status was with moderate gingivitis (gums are red in color, moderate swelling of gums) it categorized to group moderate and if it was with severe gingivitis (marked redness of gums, swelling of gums, ulcerations ) it categorized in group poor oral health. 
Patients who didn't live in Mashhad, but have been referred for deciduous teeth extraction were excluded from this study. Data were analyzed by SPSS software 11.5 version. Normality of quantitative data was tested by One Sample K.S. test. Relation of qualitative variables was analyzed by Chi-Square and difference of quantitative variables between sub-groups assessed by T-Test and ANOVA or Mann-Whitney and Kruskall-Wallis tests. Significant level was considerate less than 0.05 .

\section{Results}

In this study 254 people (totally 383 extracted teeth) participated, 140 of them were female (55\%). Mean age of participants was $39.3 \pm 13.8$ years, highest frequency was in ages 20 - 30 (24.8\%). Table 1 shows frequency of participants based on sex and age groups. Females were 6.1 years younger than males according to their mean ages $(P=0.001)$. In 115 participants $(45.3 \%)$ teeth were extracted from maxilla, 124 participants $(49.2 \%)$ from mandible and 14 (5.5\%) from both.

Reasons of TE were determined; including dental caries, periodontal diseases, to wear denture (prosthodontic), orthodontic treatment plan, non-occlusion teeth, participants`request (when patients wish to extract teeth due to economic issues despite available simple treatment), fracture and other causes. The main cause of TE was dental caries (55.1\%), following by impaction, non-occlusion tooth, prosthodontic, periodontal. There was not significant relationship between gender and reasons of TE $(P=0.3)$. Caries was most common cause of TE in both sexes. Second cause was prosthodontic in men (14\%) and impaction in women (17.1\%). Table 2 shows frequency of reason of TE in participants and Participants; characteristics are defined in Table 3.

Table 1. Frequency of participants based on sex and age groups.

\begin{tabular}{ccccccc}
\hline Sex & \multicolumn{2}{c}{ Male } & \multicolumn{2}{c}{ Female } & \multicolumn{2}{c}{ Total } \\
\hline Age group & Number & Percent & Number & Percent & Number & Percent \\
\hline$<20$ & 2 & 1.8 & 11 & 7.9 & 13 & 5.3 \\
$20-29$ & 25 & 21.9 & 38 & 27.1 & 63 & 24.8 \\
$30-39$ & 24 & 21.1 & 28 & 20 & 52 & 20.5 \\
$40-49$ & 21 & 18.4 & 33 & 23.6 & 54 & 21.3 \\
$50-59$ & 25 & 21.9 & 25 & 17.9 & 50 & 19.7 \\
$\geq 60$ & 17 & 14.9 & 5 & 3.6 & 22 & 8.7 \\
Total & 114 & 100 & 140 & 100 & 254 & 100 \\
\hline
\end{tabular}

Table 2. Different reasons of tooth extraction regard to participants' sex.

\begin{tabular}{|c|c|c|c|}
\hline Sex Reasons of Tooth Extraction & Male Number (\%) & Female Number (\%) & Total Number (\%) \\
\hline Caries & $62(54.4 \%)$ & $78(55.7 \%)$ & $140(55.1 \%)$ \\
\hline Periodontal & $10(8.8 \%)$ & $9(6.4 \%)$ & $19(7.5 \%)$ \\
\hline Prosthodontic & $16(14 \%)$ & $13(9.3 \%)$ & 29 (11.4\%) \\
\hline Impaction & $12(10.5 \%)$ & $24(17.1 \%)$ & $36(14.2 \%)$ \\
\hline Non-occlusion & $7(6.1 \%)$ & $10(7.1 \%)$ & $17(6.7 \%)$ \\
\hline Orthodontic & $1(0.9 \%)$ & $1(0.7 \%)$ & $2(0.8 \%)$ \\
\hline Fracture & $1(0.9 \%)$ & $1(0.7 \%)$ & $2(0.8 \%)$ \\
\hline Patient`s request & $4(3.5 \%)$ & $2(1.4 \%)$ & $6(2.4 \%)$ \\
\hline Others & $1(0.9 \%)$ & $2(1.4 \%)$ & $3(1.2 \%)$ \\
\hline
\end{tabular}


Table 3. Demographic characteristics of participants.

\begin{tabular}{|c|c|c|c|}
\hline & \multirow{2}{*}{ Sex } & Female & \multirow{2}{*}{$\begin{array}{c}\text { Male } \\
\text { Number (percent) }\end{array}$} \\
\hline & & Number (percent) & \\
\hline \multirow{5}{*}{ Job categories } & Student & $14(10)$ & $9(7.9)$ \\
\hline & Housewife & $115(82.1)$ & $0(0)$ \\
\hline & Employee & $5(3.6)$ & $13(11.4)$ \\
\hline & Self-employed & $6(4.3)$ & $74(64.9)$ \\
\hline & Retired & $0(0)$ & $18(15.8)$ \\
\hline \multirow{4}{*}{ Educational group } & Illiterate & $17(7)$ & $17(7)$ \\
\hline & Primary school & $64(25)$ & $44(17)$ \\
\hline & High school \& diploma & $41(16)$ & $37(15)$ \\
\hline & College degree & $18(7)$ & $16(6)$ \\
\hline \multirow{3}{*}{ Oral hygiene } & Good & 55 (39.3) & 35 (30.7) \\
\hline & moderate & 47 (33.6) & 33 (28.9) \\
\hline & Poor & 38 (27.1) & $46(40.4)$ \\
\hline \multirow{5}{*}{ Underlying disease } & None & $103(73.6)$ & $86(75.4)$ \\
\hline & Heart disease & $18(15.8)$ & $16(11.5)$ \\
\hline & diabetes & $2(1.8)$ & $5(3.6)$ \\
\hline & Heart disease \& diabetes & $0(0)$ & $6(4.3)$ \\
\hline & Other diseases & $8(7.1)$ & $10(8.1)$ \\
\hline
\end{tabular}

Periodontal diseases and prosthodontic showed most number of teeth extraction in the group of 50 - 60 years old, $63.2 \%$ and $41.4 \%$ respectively. Impaction and non-occlusion showed most number of teeth extraction in the group of 20-30 years old respectively.

The mean number of extracted teeth in current visit was $1.4 \pm 0.7$ which did not has significant difference between two sex $(P=0.8)$. There was significant difference between mean number of extracted teeth and oral hygiene groups. Participants in poor oral hygiene had more than extracted teeth compared to good and moderate oral hygiene group. But between good and moderate oral hygiene groups significant difference was not shown $(P=0.01)$.

The median of tooth brushing per day was one times (range 0 - 3) and for sugar intake was 3 times per day (range 0 - 9). There was significant difference between average of tooth brushing times in men (0.6) compared with women (1.1) $(P=0.03)$, but difference was not shown in number of sugar intake in both sex $(P=0.1)$.

The main causes of TE in participants with poor oral hygiene were dental caries (52.6\%), prosthodontic (21.1\%) and periodontal diseases, respectively. In good and moderate oral hygiene, caries (56.9\%), impaction (23.5\%) and non-occlusion (7.8\%) were common causes of TE respectively $(P<0.001)$.

In all educational groups, except those with college degrees, dental caries were the main cause of tooth extraction. In participants with college degrees, the impaction reason (44.1\%) took place before dental caries (29.4\%), $(P<0.001)$. Increase in the level of education, had significant relation with good oral hygiene $(P=$ 0.03). To compare frequency of tooth brushing in different educational level, Kruskall-Wallis test indicated that tooth brushing was 1.2 times lower in participants without college degrees $(P=0.01)$, but there was no significant difference in frequency of sugar intake between different educational levels $(P=0.29)$.

In terms of oral health status, 90 (35.5\%) of participants had high, 80 (34.5\%) and 84 (33\%), moderate and poor oral hygiene. Oral health status was better in women $(P=0.03)$.

There was significant difference in oral hygiene status between employed and unemployed women $(P=0.04)$, whereas was not in men $(P=0.89)$. 56.5\% of employed women, had poor oral hygiene, whereas $41.9 \%$ of un- 
employed women had desired oral status. Relation between dental caries and employment was indicated only in men $(P=0.01$ vs. 0.56$)$.

$20.6 \%$ of all participants were single and $79.4 \%$ were married. The median for number of children in married participants was 2 with range of 0-9. In high frequent ages group (20-30 years old), marriage did not have relation with oral health status and cause of TE $(P=0.52, P=0.78)$.

There was significant difference between mean age and frequency of tooth brushing per day in participants with TE because of "caries, periodontal and prosthodontic diseases" compared with other reasons $(P<0.001)$. It was shown that in three groups, the mean age of were 10.2 years more than other groups, tooth brushing was 0.8 times less than the others.

Thirty-five participants (13.8\%) suffered from heart diseases and 13 participants (5.1\%) had diabetes. In both cardiac and diabetic patients, dental caries were the most common cause of TE. These two diseases had association with oral health status, whereas in patients with and without mentioned disorders, the prevalence of poor oral hygiene was $47.9 \%$ and $29.6 \%$ respectively.

\section{Discussion}

In this study the most common cause of TE was dental caries, and it is pointing that in spite of improving health indexes in Iran, poor oral hygiene remains a problem that requiring more interest. Tooth decay was the most common cause of permanent TE in developing countries including India, Pakistan, Bangladesh, Libya, Nigeria, Jordan and Brazil [15]-[21]. In studies which conducted in developing countries with higher life expectancy, prevalence rate of caries and periodontal diseases as a result of tooth extraction, come closer [22].

In an investigation carried out in Iran, in 2008, the main causes of TE were non-repairable caries (32.5\%) [14]. In a study conducted in Tehran School of Dentistry in 2000, there was wide range between caries and periodontal diseases prevalence (50\% vs. 16.6\%) [8]. It should be noted that Jafarian in 2013 found the same results in Tehran [23].

The mean age of participants was 40; also ages of women were less than men. With increase in life expectancy up to more than 70 years old in Iranian men and women, the age of TE especially in women is really younger than expected.

In this study tooth extraction as a result of periodontal diseases and prosthodontic found the most in 50 - 60 ages group, whereas impaction and non-occlusion were the main reasons in ages group of 20 - 30. A Japanese study showed that women have lost their teeth because of tooth decay in a greater amount than men. Also it was shown that the dental caries in people over the age of sixteen years and periodontal disease in men of the ages group of 40 - 65 years were the main causes of TE [24].

In Bangladesh studies have shown significant relation between caries-related extraction and age, diet, dental plaque control and pattern of visiting dentist and periodontal-related extraction had relationship with age, educational level, smoking, plaque control and using mouthwash [15].

Impaction following by dental caries was the main reason for teeth extraction in participants with college degrees. Increasing in education level had association with desirable hygiene. Frequency of tooth brushing was more in educated people, but there was not significant difference between frequency of sugar intake and level of education. Unlike current study, there was no significant relation between the average number of extracted teeth and educational level in Tabriz study [9].

There was no difference in sugar intake among both sexes, also frequency of tooth brushing and oral health were in higher range in women, and they had extracted teeth in younger ages and also had higher number of extracted teeth in a single visit. We did not find difference between marital statuses, reasons of extraction and oral health status. It seems assessment of reasons for tooth decay in women, behavioral and dietary habits, in females may be considered in future studies. Life style change, higher sugar intake, women's employment, job stress and unhealthy snacks must be highlighted in future studies.

\section{Conclusion}

Tooth decay is the main reason of TE in all ages especially under the age of forty. Despite decreasing trend in risk factors of dental caries (e.g. number of pregnancies, labors and milk feeding in Iranian women), there are so many women in younger age than men referred due to caries. Poor oral health status is notable in this study. Educational level improves oral hygiene and times of tooth brushing, without any change in times of eating sug- 
ar. Community-wide education about lower consumption of sugar and to practice good oral hygiene by changing diet and improving life expectancy which requires preservation of permanent teeth until old age appears to be essential.

\section{Limitation}

Participants of this study were selected from governmental clinic, which may be not generalized to patients of private centers because of different in socio-economic level.

\section{Acknowledgements}

This study was based on a research grant from Mashhad University of Medical Sciences.

\section{References}

[1] Petersen, P.E. and Kwan, S. (2011) Equity, Social Determinants and Public Health Programmes-The Case of Oral Health. Community Dentistry and Oral Epidemiology, 39, 481-487. http://dx.doi.org/10.1111/j.1600-0528.2011.00623.x

[2] Richards, D. (2013) Oral Diseases Affect Some 3.9 Billion People. Evidence-Based Dentistry, 14, 35. http://dx.doi.org/10.1038/sj.ebd.6400925

[3] Somsak, K. and Kaewplung, O. (2014) The Effects of the Number of Natural Teeth and Posterior Occluding Pairs on the Oral Health-Related Quality of Life in Elderly Dental Patients. Gerodontology, 13, 123-128.

[4] Yu, S.J., Chen, P. and Zhu, G.X. (2013) Relationship between Implantation of Missing Anterior Teeth and Oral Health-Related Quality of Life. Quality of Life Research: An International Journal of Quality of Life Aspects of Treatment, Care and Rehabilitation, 22, 1613-1620. http://dx.doi.org/10.1007/s11136-012-0314-4

[5] Kato, M.T. and Buzalaf, M.A. (2012) Iron Supplementation Reduces the Erosive Potential of a Cola Drink on Enamel and Dentin in Situ. Journal of Applied Oral Science: Revista FOB, 20, 318-322. http://dx.doi.org/10.1590/S1678-77572012000300004

[6] Kim, J.W., Jang, K.T., Lee, S.H., Kim, C.C., Hahn, S.H. and Garcia-Godoy, F. (2001) In Vivo Rehardening of Enamel Eroded by a Cola Drink. ASDC Journal of Dentistry for Children, 68, 122-124.

[7] Lussi, A., Jaeggi, T. and Zero, D. (2004) The Role of Diet in the Aetiology of Dental Erosion. Caries Research, 38, 1, 34-44. http://dx.doi.org/10.1159/000074360

[8] Ramezanian, M. and Alizade, A. (2004) Evaluation of the Reasons for the Extraction among Patients Referred to the Oral Surgery Department, Faculty of Dentistry, Tehran University of Medical Sciences. Journal of Dental Medicine, 17, 86-90.

[9] Yazdani, J., Khashabi, E. and Ghavimi, M. (2008) Evaluation of the Reasons for the Extraction among Patients Referred to the Oral Surgery Department, Faculty of Dentistry, Tabriz University of Medical Sciences between June and February 2005. Medical Journal of Tabriz University of Medical Sciences, 30, 139-142.

[10] Gilbert, G.H., Duncan, R.P. and Shelton, B.J. (2003) Social Determinants of Tooth Loss. Health Services Research, 38, 1843-1862. http://dx.doi.org/10.1111/j.1475-6773.2003.00205.x

[11] Astrom, A.N., Ekback, G., Ordell, S. and Unell, L. (2011) Socio-Behavioral Predictors of Changes in Dentition Status: A Prospective Analysis of the 1942 Swedish Birth Cohort. Community Dentistry and Oral Epidemiology, 39, 300-310. http://dx.doi.org/10.1111/j.1600-0528.2010.00594.x

[12] Kida, I.A., Astrom, A.N., Strand, G.V. and Masalu, J.R. (2006) Clinical and Socio-Behavioral Correlates of Tooth Loss: A Study of Older Adults in Tanzania. BMC Oral Health, 6, 5. http://dx.doi.org/10.1186/1472-6831-6-5

[13] Hassan, A.K. (2000) Reasons for Tooth Extraction among Patients in Sebha, Libyan Arab Jamahiriya: A Pilot Study. Eastern Mediterranean Health Journal [La revue de sante de la Mediterranee orientale] [al-Majallah al-sihhiyah li-sharq al-mutawassit], 6, 176-178.

[14] Peimani, A. and Bakhshi, H. (2011) Assessment of the Reasons for Tooth Extraction among Patients Referred to the Rafsanjan Dental School (2008). Journal of Rafsanjan University of Medical Sciences and Health Services, 10, 94-102.

[15] Akhter, R., Hassan, N.M., Aida, J., Zaman, K.U. and Morita, M. (2008) Risk Indicators for Tooth Loss Due to Caries and Periodontal Disease in Recipients of Free Dental Treatment in an Adult Population in Bangladesh. Oral Health \& Preventive Dentistry, 6, 199-207.

[16] Anand, P.S., Kamath, K.P. and Nair, B. (2010) Trends in Extraction of Permanent Teeth in Private Dental Practices in Kerala State, India. The Journal of Contemporary Dental Practice, 11, 41-48. 
[17] Ashiwaju, M.O., Folayan, M.O., Sote, E.O. and Isikwe, M.C. (2011) Pattern of Tooth Extraction in Children Attending Tertiary Health Care Centers in Nigeria: A Prospective Study. The Journal of Clinical Pediatric Dentistry, 36, 107-110. http://dx.doi.org/10.17796/jcpd.36.1.3616j76286616672

[18] Byahatti, S.M. and Ingafou, M.S. (2011) Reasons for Extraction in a Group of Libyan Patients. International Dental Journal, 61, 199-203. http://dx.doi.org/10.1111/j.1875-595X.2011.00057.x

[19] Caldas Jr., A.F. (2000) Reasons for Tooth Extraction in a Brazilian Population. International Dental Journal, 50, 267-273. http://dx.doi.org/10.1111/j.1875-595X.2000.tb00564.x

[20] Haseeb, M., Ali, K. and Munir, M.F. (2012) Causes of Tooth Extraction at a Tertiary Care Centre in Pakistan. Journal of the Pakistan Medical Association, 62, 812-815.

[21] Quteish Taani, D.S. (2003) Periodontal Reasons for Tooth Extraction in an Adult Population in Jordan. Journal of Oral Rehabilitation, 30, 110-112. http://dx.doi.org/10.1046/j.1365-2842.2003.00981.x

[22] Ong, G., Yeo, J.F. and Bhole, S. (1996) A Survey of Reasons for Extraction of Permanent Teeth in Singapore. Community Dentistry and Oral Epidemiology, 24, 124-127. http://dx.doi.org/10.1111/j.1600-0528.1996.tb00828.x

[23] Jafarian, M. and Etebarian, A. (2013) Reasons for Extraction of Permanent Teeth in General Dental Practices in Tehran, Iran. Medical Principles and Practice, 22, 239-244. http://dx.doi.org/10.1111/j.1600-0528.1996.tb00828.x

[24] Morita, M., Kimura, T., Kanegae, M., Ishikawa, A. and Watanabe, T. (1994) Reasons for Extraction of Permanent Teeth in Japan. Community Dentistry and Oral Epidemiology, 22, 303-306. http://dx.doi.org/10.1111/j.1600-0528.1994.tb02056.x 